\title{
ASSESSMENT OF THE LOCAL SAR DISTORTION BY MAJOR ANATOMICAL STRUCTURES IN A CYLINDRICAL NECK PHANTOM
}

Paulides MM, Wielheesen DHM, Van der Zee J, Van Rhoon GC

Erasmus MC - Daniel den Hoed Cancer Center, Department of Radiation Oncology, Section Hyperthermia PO box 5201, NL-3008 AE, Rotterdam, The Netherlands

Tel.no. : +31 104391676

Fax.no. : +31 104391022

E-mail: M.Paulides@ErasmusMC.nl

August 22, 2007

Running title: "Parameter investigation into heating neck tumours" 


\begin{abstract}
The objective of this work is to gain insight in the distortions on the local SAR distribution by various major anatomical structures in the neck. High resolution 3D FDTD calculations based on a variable grid are made for a semi-3D generic phantom based on average dimensions obtained from CT-derived human data and in which simplified structures representing trachea, cartilage, spine and spinal cord are inserted. In addition, phantoms with dimensions equal to maximum and minimum values within the CT-derived data are also studied. In all cases, the phantoms are exposed to a circular coherent array of eight dipoles within a water bolus and driven at $433 \mathrm{MHz}$. Comparisons of the SAR distributions due to individual structures or a combination of structures are made relative to a cylindrical phantom with muscle properties.

The calculations predict a centrally located region of high SAR within all neck phantoms. This focal region, expressed as contours at either $50 \%$ or $75 \%$ of the peak SAR, changes from a circular cross-section in the case of the muscle phantom to a donut shaped region when the anatomical structures when the anatomical structures are present. The presence of the spine causes the greatest change in the SAR distribution, followed closely by the trachea. Global changes in the mean SAR relative to the uniform phantom are $<11 \%$ whilst local changes are as high as 2.7-fold. There is little difference in the focal dimensions between the average and smallest phantoms but a decrease in the focal region is seen in the case of the largest phantom.

This study presents a first step towards understanding of the complex influences of the various parameters on the SAR pattern which will facilitate the design of a site-specific head and neck hyperthermia applicator.
\end{abstract}

Key words: Semi-deep hyperthermia, FDTD modelling, SAR, antenna array, head and neck tumours, parameter study.

\title{
${ }_{25} \quad 1 \quad$ Introduction
}

Patients with advanced carcinomas in the head and neck (H\&N) have a dismal prognosis and locoregional control for this site still poses a major therapeutic challenge. Combining radiotherapy (RT) with simultaneous chemotherapy improves the treatment outcome but at the cost of increased toxicity. Triggered by the phase III trial of Valdagni et al. [1] [2], we started the exploration of hyperthermia (HT) applied to the head and neck region. Valdagni demonstrated that with a nonspecific HT-applicator already a significant increase in local control (from $24 \%$ for RT alone versus $69 \%$ for RT plus HT) is achieved for metastatic lymphnodes in Stage IV carcinoma of the H\&N, without additional toxicity. However, for an optimal HT treatment of the primary tumour and metastatic lymphnodes a specific H\&N HT applicator is warranted. In our opinion such a site specific designed, multi-element, applicator would also enable a substantial improved control of the specific absorption rate (SAR) deposition pattern and is expected to result in a higher quality of the HT-treatment (Wust et al. [3]).

The head and neck region is characterized by many tissue transitions, small in diameter structures and large cooling vessels. Applying an adequate thermal dose to the head and neck region therefore is a challenging task. In the clinical setup the thermal dose is determined by a huge amount of parameters with separate and combined influences. From a designers point of view 
the aim is to approach the problem by simplification of the problem since this increases the development progress, provides a better understanding of separate parameter influences, enables verification measurements and links with quality assurance setups. As a first step we start this SAR based investigation with the most simple setup: an array of eight dipole antennas radiating 5 a centrally positioned muscle equivalent cylinder representing a first approximation of the neck. This simple setup forms the reference configuration from which the problem is extended to a more and more realistic neck anatomy. The influence on the SAR pattern of various parameters can now be seen as distortions on the local SAR pattern with respect to the reference configuration.

The causes of the perturbations on the SAR distribution in the region of interest are clustered in three groups: 1) anatomy, 2) antenna array and 3) waterbolus related distortions. These parameters can be investigated independently allowing a better understanding of their, sometimes opposing, effects. In this publication we dedicate our attention to the anatomy related effects, thus a fixed applicator and waterbolus design are chosen, i.e. an array of dipole antennas embedded in an infinite water space. The SAR distribution in the neck is, to a large amount, dictated by

15 the tissue distribution in the $\mathrm{H} \& \mathrm{~N}$ region and it is our expectation that a thorough exploration of the anatomy based distortions will pay off in the applicator design. Exploring all different anatomy related influences is a vast task and therefore a distinction is made between $2 \mathrm{D}$ and $3 \mathrm{D}$ anatomical shape related SAR perturbations. In this work we focus on $2 \mathrm{D}$ related effects and the $3 \mathrm{D}$ related distortions are subject of further research. With $2 \mathrm{D}$ related distortions we refer to the

20 influences on the SAR pattern caused by differences in tissue in the radial direction, however the assessment is carried out with a 3D FDTD calculation. The investigation is carried out by placing inserts representing major anatomical structures in the muscle cylinder. The distortions on the SAR pattern caused by these major structures are subsequently addressed by comparison of their respective SAR distribution with the SAR pattern in the muscle cylinder.

25 This investigation is carried out at $433 \mathrm{MHz}$ since it is found to be within the range of promising frequency candidates for a head and neck applicator [4] and equipment for latter verification measurements at this frequency is available at our clinic. The configurations are chosen such that at $433 \mathrm{MHz}$ in a water environment $\left(\epsilon_{r}=78\right)$ the cylindrical phantoms are located in the far-field region of the antenna array. This, combined with a contrast type SAR pattern distortion evaluation

so parameter, i.e. Relative Differences (RD's), yields an antenna (pattern) invariant investigation.

\section{Materials and Methods}

\subsection{Creation of the average neck phantom}

An homogeneous muscle cylinder is used to model the anatomy as a starting point. From this base situation the setup is extended towards a more realistic neck anatomy by inclusion of inserts

35 representing major structures, e.g. trachea and spine. The shapes of the structures in the neck vary strongly in the radial direction and only to a lesser extent in the axial direction. Therefore the shape of these structures is approximated by elementary shapes (figure 1). These are extruded resulting in semi-3D approximations of the original structures. The dimensions of the shapes are defined using Computerized Tomography (CT)-scans from which the minimum, average and 
Table 1: Relative permittivity, conductivity and mass density for the selected neck structures and the surrounding water at $433 \mathrm{MHz}$.

\begin{tabular}{|l|c|c|c|}
\hline Structure & $\epsilon_{r}$ & $\sigma(S / m)$ & $\rho\left(\mathrm{kg} / \mathrm{m}^{3}\right)$ \\
\hline \hline Muscle & 57 & 0.80 & 1050 \\
Trachea (air) & 1 & 0.00 & 1 \\
Cartilage* $^{*}$ & 45 & 0.60 & 1100 \\
Spine (Bone, cortical) & 13 & 0.09 & 1595 \\
Spinal cord (Brain, white matter) & 42 & 0.45 & 1050 \\
\hline \hline De-ionised water $\left(0.00 \mathrm{~g} / \mathrm{l} \mathrm{NaCl,} \mathrm{T}=20^{\circ} \mathrm{C}\right)$ & 78 & 0.04 & 998 \\
\hline \multicolumn{2}{|c|}{$*$ no discrimination is made between dry and wet cartilage }
\end{tabular}

maximum dimensions and locations of the major neck structures are extracted (see section 2.1.1). The neck in this case is defined as the elliptical shaped area between the widening curvatures towards the head and towards the shoulders.

In this parameter study we focus on structures with an expected large influence on the SAR 5 pattern. The selection criteria for inclusion in the model of an anatomical structure are: 1) existence in the neck in the axial plane (from collar bone up to the oropharynx), 2) amount of dielectric contrast with neighbouring tissue (muscle), 3) size of its transverse cross-section, 4) amount of perfusion. It is expected that the amount of perfusion is an issue mainly in areas consisting of cartilage. The SAR in these areas probably will not appear to be dramatic but might, due to the poor perfusion, cause limiting hotspots, i.e. burns. Table 1 shows the list of structures that are selected using these criteria. The "cartilage" is the cartilage of the trachea. The skin and oesophagus are not included in the considerations due to their small transverse cross-section and low dielectric contrast with the neighbouring muscle tissue. No fat is selected since in these patients only a small amount of fatty tissue is present, which is overshadowed by the large muscles that it surrounds. The influence of a small layer of subcutaneous fat is assessed as a side step.

\subsubsection{Determination of the elementary shapes}

The selected structures (table 1) are fitted by elementary shapes (figure 1b). The dimensions of these shapes are extracted from CT scans of eight patients from a representative patient group, i.e. $\mathrm{H} \& \mathrm{~N}$ patients at our radiotherapy department with tumours at various sites (hypopharynx, parotic gland (3x), larynx, oropharynx and nasopharynx $(2 \mathrm{x}))$.

The dimension extraction is carried out by calculation of the mean of the Hounsfield Unit (HU) distribution, i.e. averaging the HU's in the CT scans of the 4th down to the 6 th cervical vertebra for every patient (14 - 19 slices). An example average HU distribution is shown in figure 1a. Markers are positioned in this average $\mathrm{HU}$ distribution at every extent of a structure and subsequently the dimensions of the structures are calculated using their respective locations. For example, a circle is described by four markers defining two radii (in the $\mathrm{x}$ and $\mathrm{y}$-direction). These markers are then used to calculate the smallest, average and largest value of each dimension (figure 1b), which are 
presented in table 2 . These dimensions are calculated such that they are symmetrical with respect to the sagittal plane at $x=0$.

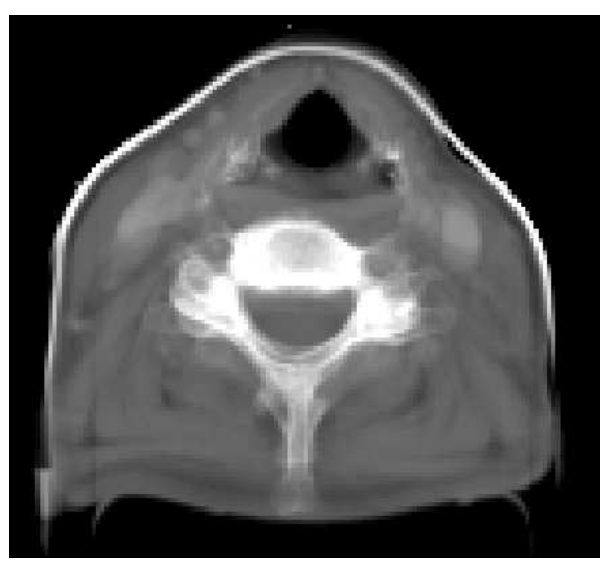

a) Example average HU distribution

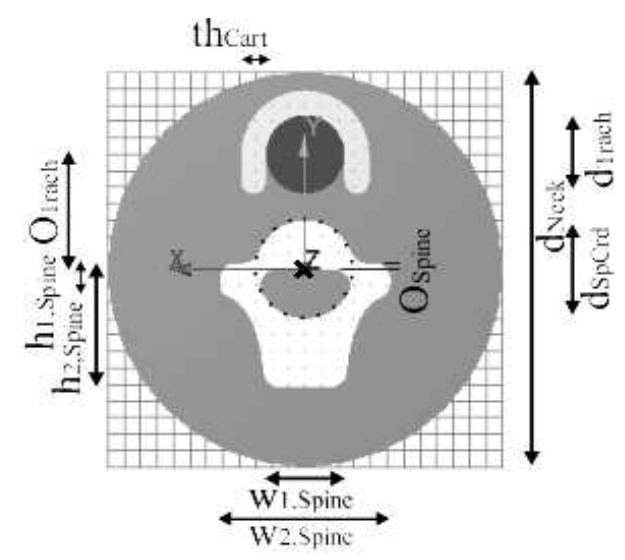

b) Rounded elementary-shape fit

Figure 1: a) Example average ( $4^{\text {th }} \leftrightarrow 6^{\text {th }}$ neck vertebra) HU distribution of one patient and b) the rounded elementary-shape fit with 2D shapes (average dimensions of multiple patients). The dimensions of the shapes correspond to those of tabel 2 and the distance between the mesh lines is $5 \mathrm{~mm}$. Note that the lower part of the spine seems to have a larger width in b) compared to a) which is the result of (i) the different shapes of the vertebra's and (ii) our focus on the central vertebra, which sometimes consists of two peaks.

Table 2: Smallest, average and largest dimensions $(\mathrm{mm})$ of the selected neck structures and the rounding radius per geometry. The abbreviations refer to a d(iameter), th(ickness), $\mathrm{O}$ (ff-set), $\mathrm{h}$ (eight), w(idth) or r(adius). The off-sets are shifts, with respect to the origin, in the y-direction.

\begin{tabular}{|l|c|c|c|}
\hline Dimension & Smallest & Average & Largest \\
\hline \hline$d_{\text {Neck }}$ & 97 & 120 & 135 \\
$d_{\text {Trach }}$ & 19 & 25 & 29 \\
$O_{\text {Trach }}$ & 28 & 36 & 41 \\
th $h_{\text {Cart }}$ & 5 & 8 & 9 \\
$d_{\text {SpCrd }}$ & 27 & 31 & 34 \\
$O_{\text {SpCrd }}$ & 0 & 0 & 0 \\
$w_{1, \text { Spine }}$ & 48 & 52 & 58 \\
$w_{2, \text { Spine }}$ & 19 & 25 & 29 \\
$h_{1, \text { Spine }}$ & 25 & 37 & 51 \\
$h_{2, \text { Spine }}$ & 8 & 10 & 12 \\
$O_{\text {Spine }}$ & 2 & 2 & 2 \\
\hline$r_{\text {rounding }}$ & 4 & 5 & 6 \\
\hline
\end{tabular}

The most difficult tissue type to assign is the cartilage surrounding the trachea, due to its 
low contrast with surrounding muscle. Therefore, for guidance of positioning the markers of the trachea surrounding cartilage, an anatomy book is used [5].

The elementary shapes are subsequently extruded using the solid modelling kernel of SEMCAD [6] to become a $250 \mathrm{~mm}$ high cylindrical shape: the "solid model". This solid model is rounded, 5 using rounding radius $r_{\text {rounding }}$, to avoid local hotspots due to a sharp model. This rounding is applied because the aim is to study macroscopic influences of anatomical structures and not hotspots due to patient specific sharp edged structures. Further, applying the rounding increases the visual resemblance between the model and the average HU distributions. The neck model is chosen sufficiently high to avoid influences, in the plane of interest, of the upper and lower

10 transitions (tissue $\leftrightarrow$ water). A cross-section of the average solid model is shown in figure $1 \mathrm{~b}$. Similar setups are conducted using the smallest and largest found dimensions (table 2) forming the "smallest" and "largest" cylindrical neck phantoms.

\subsubsection{Creation of a semi-3D neck anatomy for contour verifications}

A semi-3D neck anatomy is created to be able to investigate the difference in SAR distribution

15 of an approximation of the neck by 1) a cylindrical neck shape with elementary shaped inserts versus 2) a neck with actual contours. This latter anatomy is constructed from one slice, at the height of the 5th cervical vertebra, of one patient. The patient with neck dimensions close to average (table 2) is selected. Note that this does not mean that every dimension of the patients neck structures is average.

${ }_{20}$ The contours of the neck structures are found by applying a HU-based threshold contour fit procedure. These contours are subsequently extruded to form a semi-3D neck anatomy, where the same structures are selected as for the average neck phantom (table 1). There was little difference in HU value between cartilage and the surrounding muscle so only a small piece of (probable ossified) cartilage could be selected. It is noted that simulations, with assigning bone and subsequent

25 cartilage to this small piece, have confirmed that its influence is negligible (in the $\mathrm{z}=0 \mathrm{~cm}$ plane) so the small piece of cartilage was excluded for further calculations.

\section{$2.2 \quad$ Applicator model}

The applicator is modelled by, a close to optimum number of [7], eight dipole antennae, radiating at $433 \mathrm{MHz}$, that are positioned equidistantly on an imaginary ring surrounding the neck (figure 2a).

30 The dipoles are embedded in an infinite water space because we anticipate the use of a waterbolus however the disturbing influences of the waterbolus on the SAR pattern are outside the scope of this study.

The diameter of the applicator ring is chosen such that it can enclose a large male neck $(\mathrm{d}=$ $16 \mathrm{~cm})$ with a sufficient overlap $(>\lambda / 2=4 \mathrm{~cm})$ at all sides, i.e. the diameter of the antenna ring 35 is chosen to be $25 \mathrm{~cm}$. Halve-wave dipoles, resonant at $433 \mathrm{MHz}$, are utilized in the antenna ring. In practice, a somewhat smaller height than $\lambda / 2$ must be used for the dipoles to account for their, non-infinitely small $(0.25 \mathrm{~cm})$, diameter [8]: the height of the dipoles is $3.25 \mathrm{~cm}$. The radiation pattern of the antennas is not influenced by the phantom because the phantom is located in the far 
field $(\sim 4 \mathrm{~cm}[8])$. Therefore, the choice of applicator antennas, and thus their radiation pattern, is in fact of limited importance since a contrast type of evaluation is used.

\section{$2.3 \quad$ FDTD modelling}

For the calculations of the electromagnetic field and SAR distribution we utilized SEMCAD [6]:

5 a Finite Difference Time Domain (FDTD) [9] algorithm. For the FDTD algorithm, the entire computational domain must be divided in voxels, i.e. small brick shaped elements. The computational domain is truncated using Highdon's $2^{\text {nd }}$ order boundary conditions [9] and steady state is assumed after 10 periods. The solid model of the applicator and tissue are used to assign a label to each voxel, in this way describing the setup in voxels. Every label is assigned certain dielectric

10 properties. The dielectric properties of the structures in the neck are found using an application of IFAC-CNR [10] based on a parametric model by Gabriel et al. [11] (table 1).

The dielectric properties of the trachea are chosen equal to those of air. The properties of the de-ionised water (salinity $=0.00 \mathrm{~g} / \mathrm{l}, \mathrm{T}=20^{\circ} \mathrm{C}$ ) in the waterbolus are found in both a publication of Stogryn [12] and of the ESHO taskgroup comittee [13]. The properties of a voxel in the $\mathrm{x}, \mathrm{y}$ and z-direction are equal so an isotropic tissue distribution is obtained.

SEMCAD provides the possibility of a global grid refinement: the grid lines do not have to be spaced homogeneously. This enables the modelling of fine structures whereas the computational effort is only gradually higher. In literature a maximum of the grid stepping between $\lambda / 15$ [9] and $\lambda / 20$ is indicated for sufficient accuracy for a variable grid. This is most critical in regions with high permittivities, which in this case means in the water environment. For this research the maximum grid step, far from the region of interest, is $5 \mathrm{~mm}$ thus the grid steps remains smaller than $\lambda / 15: \Delta_{\max }<\lambda / 15$. For the main investigation setup and the setup to verify the contour influences the respective grid steps are given below.

Neck phantoms (smallest, average and large) The region of interest, i.e. the neck slice between the antenna elements, of the cylindrical neck phantom is modelled with grid steps of $1 \mathrm{~mm}$ in the $\mathrm{x}$ and $\mathrm{y}$-direction (see figure $2 \mathrm{~b}$ ), however at the antennas a local refinement is applied. The grid steps in tissue in the z-direction vary from 0.5 to $5 \mathrm{~mm}$, but the maximum grid step in the region of interest is $2 \mathrm{~mm}$.

Semi-3D neck anatomy In the z-direction the grid steps vary from 0.5 to $5 \mathrm{~mm}$ but the grid 30 steps are $2.5 \mathrm{~mm}$ in the $\mathrm{x}$ and $\mathrm{y}$-direction since we only aim to compare global SAR patterns using this geometry.

The source of the dipole antennas is modelled with a hard source [9] at $433 \mathrm{MHz}$, i.e. no feeding circuits are modelled. It is positioned at the $2.5 \mathrm{~mm}$ gap between the two conducting $(\sigma=\infty)$ poles. The modelling of the antenna is carried out with space steps down to $0.5 \mathrm{~mm}$ which provides 35 accurate results as presented by Hertel et al. [18]. 


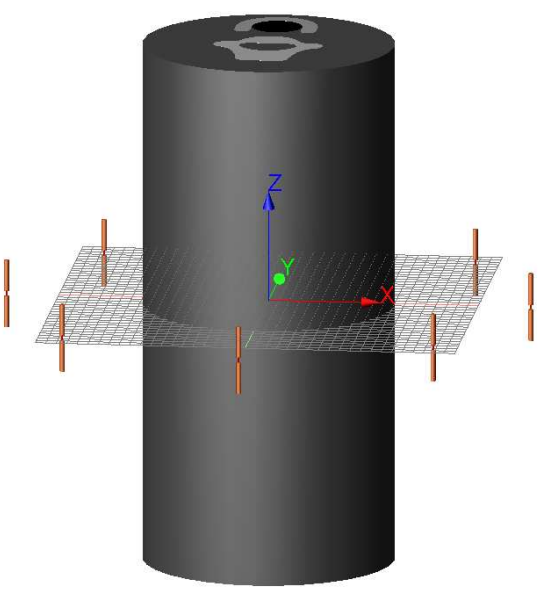

a) Antenna setup

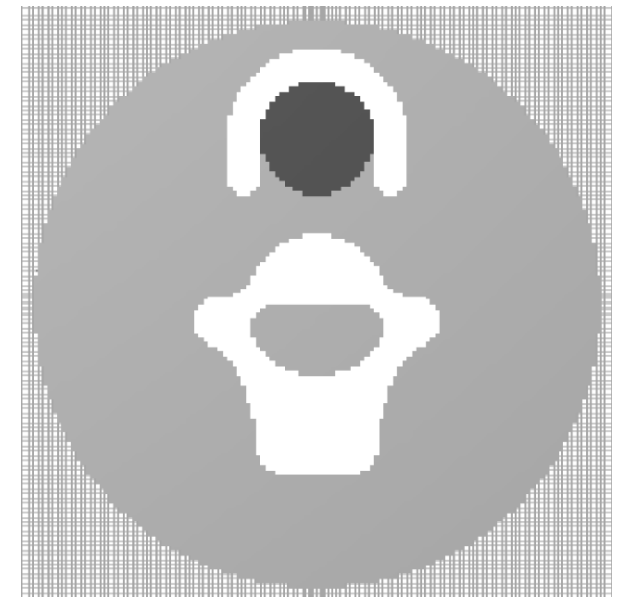

b) Average neck model

Figure 2: Visualization of a) the antenna setup surrounding the 3D solid model of the average neck phantom. and b) a cross-section at $\mathrm{z}=0 \mathrm{~mm}$ through the FDTD implementation of the average neck phantom, with an overlay of the grid lines. For clarity, the surrounding infinite water space is not visualized in both figures.

\subsection{Evaluation}

Evaluation of the SAR distributions is carried out using data of the plane at $\mathrm{z}=0 \mathrm{~cm}$ through the phantom, i.e. $\mathrm{SAR}(\mathrm{x}, \mathrm{y}, \mathrm{z}=0 \mathrm{~cm})$, since the main influences for the neck phantoms are in the $2 \mathrm{D}$ plane. A contrast type formulation is defined to be able to separately investigate the influences of 5 the geometry thus subtracting the influences of the antenna array. The Relative Difference (RD) function is defined as:

$$
R D(x, y)=\frac{S A R_{g u c}(x, y)-S A R_{r e f}(x, y)}{S A R_{r e f}(x, y)} \cdot 100 \%,
$$

where $S A R_{\text {ref }}(x, y)$ is the SAR value in the reference situation, i.e. the homogeneous muscle cylinder, and $S A R_{g u c}(x, y)$ is the SAR value in the geometry under consideration (guc). This $\mathrm{RD}$ quantifies the positive or negative influence of the anatomy on the SAR distribution, i.e. the

10 increase or decrease of the SAR by the anatomy. The minimum, mean, maximum and the standard deviation values of the RD serve as evaluation parameters:

$$
\begin{gathered}
R D_{\min }=\min \{R D(x, y)\} \\
\overline{R D}=\frac{\sum_{N} w(x, y) R D(x, y)}{\sum_{N} w(x, y)}, \quad w(x, y)=\Delta x(x, y) \Delta y(x, y) \\
\sigma=\sqrt{\frac{1}{N-1} \sum_{N}(R D(x, y)-\overline{R D})^{2}}
\end{gathered}
$$




$$
R D_{\max }=\max \{R D(x, y)\},
$$

of the distribution of the relative differences. $N$ is the total amount of voxels in the region under consideration and $\Delta x$ and $\Delta y$ are the voxel sizes of every voxel in two directions. For calculation of the mean values the weighting with $w(x, y)$ is applied to account for the variable grid. The importance of this weighting is small since the grid step differs only at the locations of

5 the antennas where refinement of the grid is applied. $R D_{\min }$ and $R D_{\max }$ are used to quantify the local SAR influences whereas $\overline{R D}$ and $\sigma$ are used to quantify the global SAR (shape) distortion.

The area enclosed by the $50 \%$ and $75 \%$ iso-SAR contours are investigated to compare intergeometry results quantitatively in terms of the change in focus size, i.e. the central region of high SAR values.

\section{${ }_{10} 3$ Results}

\subsection{Influences per structure}

First, a full analysis is carried out on the average geometry with all inserts. This clarifies the way all setups are evaluated. Each evaluation is a comparison with respect to a muscle cylinder with an identical radius. For clarity of the figures the outer tissue SAR values are not shown unless indicated otherwise.

Figure $3 \mathrm{a}$ and $3 \mathrm{~b}$ show the SAR distribution in the average muscle cylinder and average total neck anatomy at a cross-section through $\mathrm{z}=0 \mathrm{~cm}$. The SAR patterns differ mainly centrally and superficially at and near the trachea. For the total neck anatomy the SAR values are zero at the trachea due to the zero conductivity. Further, a low conductivity at the spine results in low SAR values. The central hotspot in the muscle cylinder is defocussed due to reflections at the muscle-bone transition but it remains centrally located. The high SAR values at the location of the spinal cord are lowered due to the anatomical properties. Figure $3 \mathrm{c}$ and $3 \mathrm{~d}$ visualize the RD distribution and the $\mathrm{RD}$ profile at $\mathrm{x}=0 \mathrm{~m}$ respectively. These images show that the anatomy has a positive influence on the SAR around the spine $(R D \sim 80 \%)$ and a negative influence at the spine ${ }_{25}(R D \sim-80 \%)$ and trachea $(R D=-100 \%)$. Regions with a negative $R D$ value $(\sim-25 \%)$ can also be found approximately $1 \mathrm{~cm}$ underneath the "skin". The cross-section at $\mathrm{x}=0$ is visualized to show the effect of the conductivity transitions, i.e. all the sharp edges.

Figure 4 shows the SAR distribution in the average muscle cylinder and the average muscle cylinder with various clusters of inserts. This figure shows that the high superficial SAR values near the trachea, induced by the insertion of trachea and surrounding cartilage, are reduced by the subsequent insertion of the spine and spinal cord. As a consequence the negative $\mathrm{RD}$ values near the trachea in figure $4 \mathrm{e}$ are not present in figure $4 \mathrm{f}$ and figure $4 \mathrm{~g}$. The figure also shows that the global SAR pattern is determined mainly by the cluster containing spine and spinal cord (see $35 \quad \mathrm{SAR}$ and RD distributions).

Table 3 and table 4 are given to quantitatively analyse the RD and SAR patterns. In this 

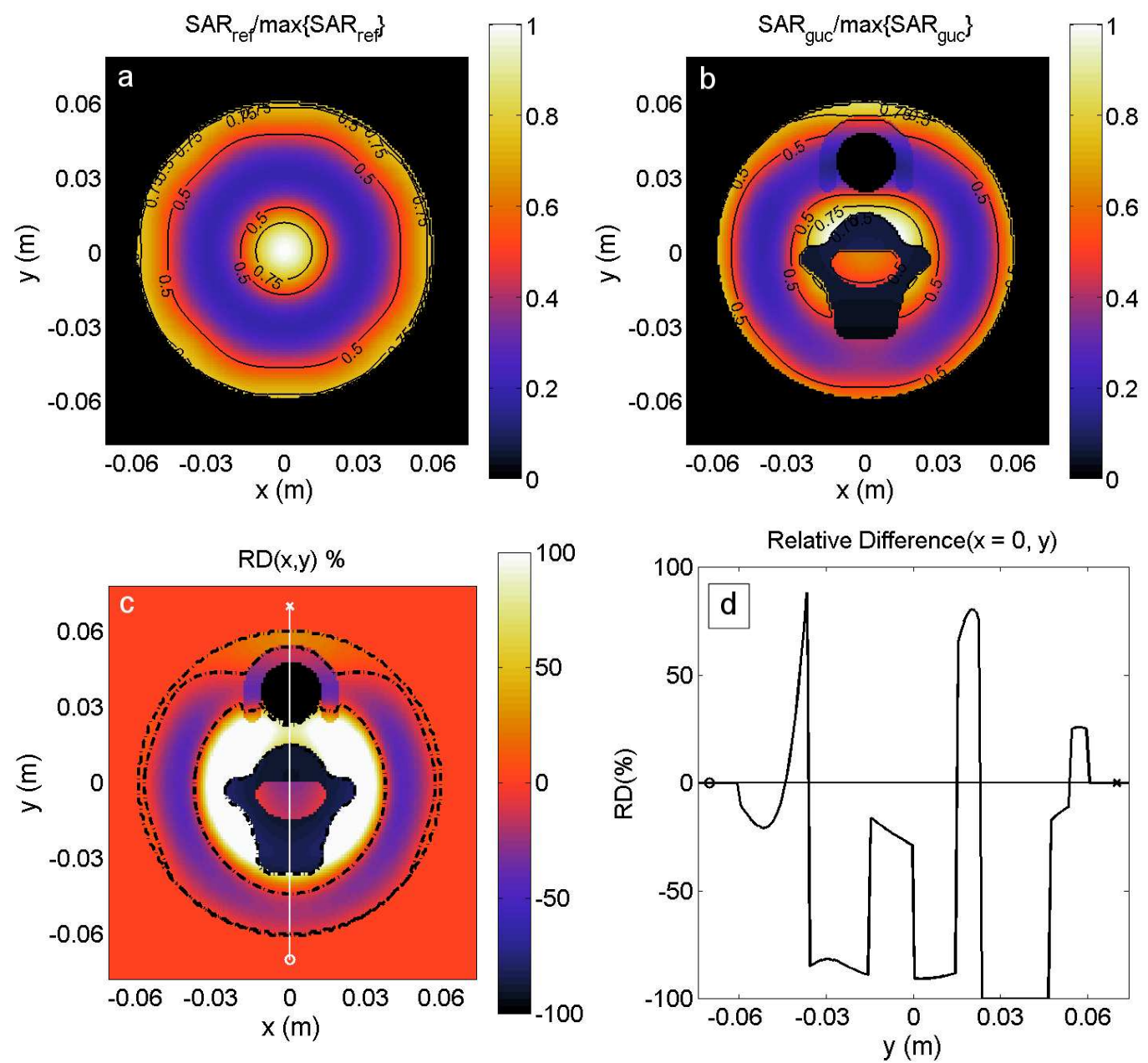

Figure 3: a) normalized SAR pattern of the muscle cylinder, b) normalized SAR pattern of the total neck anatomy, c) their RD pattern and $\mathrm{d}$ ) RD profile at $\mathrm{x}=0 \mathrm{~m}$ are visualized for the average anatomy setup at $\mathrm{z}=0 \mathrm{~m}$. The $50 \%$ and $75 \%$ iso-SAR contours are indicated in a) and b), and in c) the $\mathrm{RD}=0 \%$ contours are visualized (-.-). 


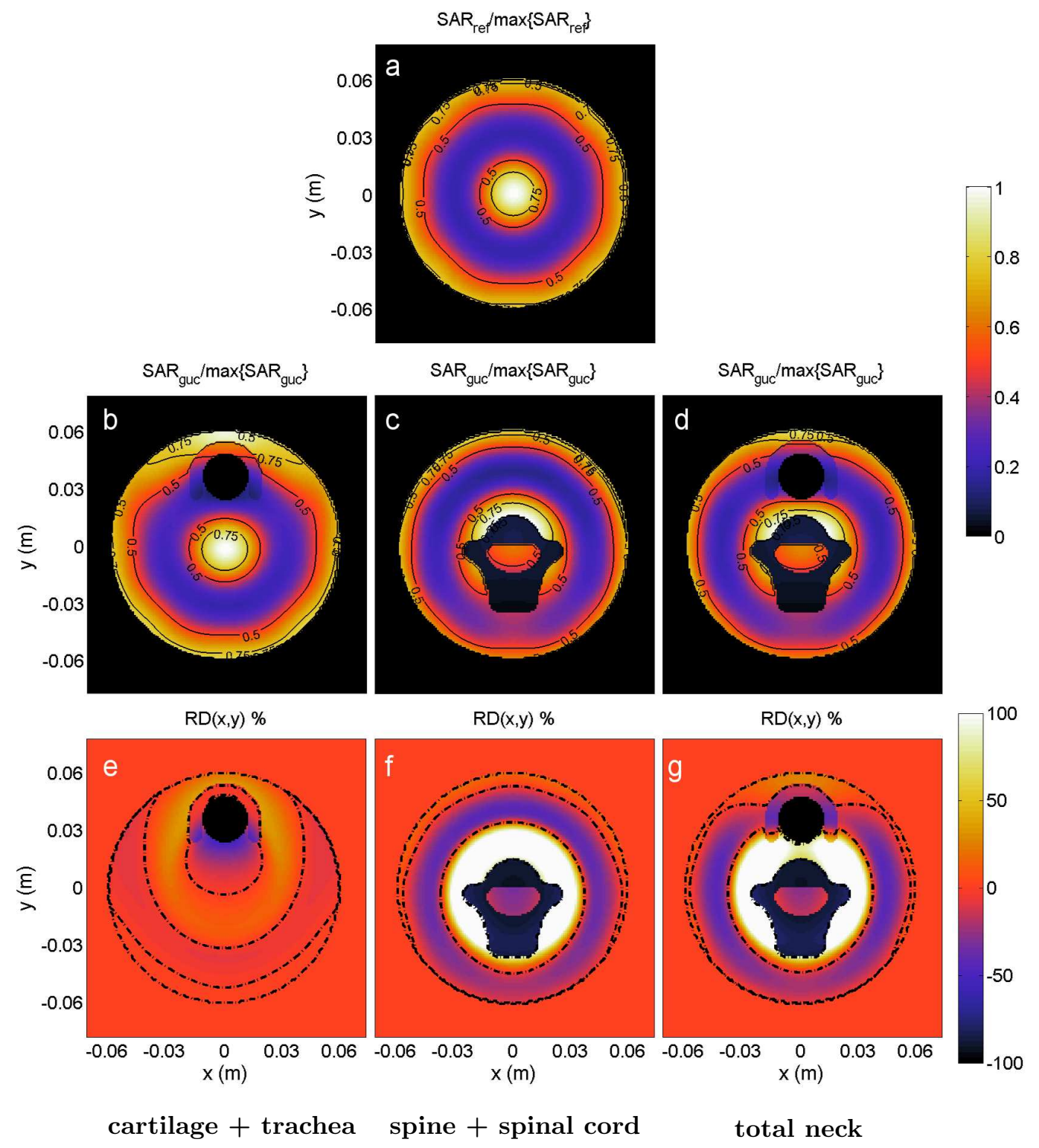

Figure 4: The normalized SAR and RD patterns at cross sections through the neck phantom at $\mathrm{z}=0 \mathrm{~m}$ are visualized for the muscle cylinder (top), the muscle cylinder with trachea and cartilage insert (left), the muscle cylinder with spine and spinal cord insert (center) and the total neck anatomy setup (right) for the average setup $\left(d_{N e c k}=12 \mathrm{~cm}\right)$. The $50 \%$ and $75 \%$ iso-SAR contours are indicated in the SAR distributions and the zero RD contours are indicated in the RD distribution (-.-). 
Table 3: $R D_{\min }, \overline{R D}, \sigma$ and $R D_{\max }$ values (\%) per geometry for setups with smallest, average and largest dimensions ( $d_{\text {Neck }}$ is $9.7,12$ or $13.5 \mathrm{~cm}$ respectively).

\begin{tabular}{|l|c|c|c|}
\hline & \multicolumn{1}{|c|}{ Smallest } & Average & Largest \\
Geometry & $R D_{\min }: \overline{R D} \pm \sigma: R D_{\max }$ & $R D_{\min }: \overline{R D} \pm \sigma: R D_{\max }$ & $R D_{\min }: \overline{R D} \pm \sigma: R D_{\max }$ \\
\hline \hline Musc+cart & $-31: 0 \pm 6: 5$ & $-33:-1 \pm 6: 7$ & $-27: 5 \pm 7: 16$ \\
Musc+trach & $-100:-1 \pm 23: 44$ & $-100:-2 \pm 23: 36$ & $-100:-2 \pm 26: 45$ \\
Musc+cart+trach & $-100:-2 \pm 23: 38$ & $-100:-3 \pm 24: 36$ & $-100:-2 \pm 27: 50$ \\
Musc+sp.crd & $-42: 0 \pm 10: 18$ & $-42: 0 \pm 10: 24$ & $-42: 0 \pm 10: 29$ \\
Musc+sp & $-92: 11 \pm 61: 124$ & $-92: 2 \pm 58: 157$ & $-92: 1 \pm 61: 190$ \\
Musc+sp.crd+sp & $-91: 11 \pm 70: 149$ & $-91: 4 \pm 69: 200$ & $-92: 3 \pm 71: 242$ \\
Total neck & $-100: 11 \pm 81: 172$ & $-100: 2 \pm 75: 224$ & $-100:-1 \pm 76: 274$ \\
\hline
\end{tabular}

Table 4: The areas $\left(\mathrm{cm}^{2}\right)$ enclosed by centrally located $50 \%$ and $75 \%$ iso-SAR contours per geometry for setups with smallest, average and largest dimensions $\left(d_{N e c k}\right.$ is $9.7,12$ or $13.5 \mathrm{~cm}$ respectively).

\begin{tabular}{|l|c|c|c|}
\hline & Smallest & Average & Largest \\
Geometry & $S A R_{50 \%}: S A R_{75 \%}$ & $S A R_{50 \%}: S A R_{75 \%}$ & $S A R_{50 \%}: S A R_{75 \%}$ \\
\hline \hline Musc & $9.6: 4.0$ & $9.6: 4.0$ & $5.2: 0.0$ \\
Musc+cart & $9.6: 4.1$ & $9.8: 4.1$ & $5.2: 0.0$ \\
Musc+trach & $9.7: 4.1$ & $9.7: 4.1$ & $4.4: 0.0$ \\
Musc+cart+trach & $9.8: 4.0$ & $9.8: 4.1$ & $3.6: 0.0$ \\
Musc+sp.crd & $8.9: 2.5$ & $8.3: 2.4$ & $3.6: 0.3$ \\
Musc+sp & $10.5: 4.4$ & $11.0: 4.7$ & $6.1: 0.0$ \\
Musc+sp.crd+sp & $14.0: 3.7$ & $13.4: 3.8$ & $4.2: 0.0$ \\
Total neck & $14.2: 4.0$ & $14.2: 3.5$ & $2.8: 0.0$ \\
\hline
\end{tabular}

section we only refer to the values belonging to the average setups. In table 3 , the $R D_{\min }, \overline{R D}$, $\sigma$ and $R D_{\max }$ values per geometry are given. From this table, for the average geometry, it can be concluded that the largest local influence is a result from the trachea (low $R D_{\min }$ ). The largest global distortion is due to the inclusion of the spine (high $\sigma$ ). The spinal cord and cartilage cause 5 relatively little distortion.

Table 4 provides the areas enclosed by the centrally located $50 \%$ and $75 \%$ iso-SAR contours per geometry. This table indicates an increased $50 \%$ SAR area for the setups with inserts, however the area within the $75 \%$ iso-SAR contour in some cases slightly decreases (because the normalization point increases). The latter effect is due to the more irregular tissue distribution at the location 10 of the high SAR values, which disturbs the Gaussian shape of the focus. 

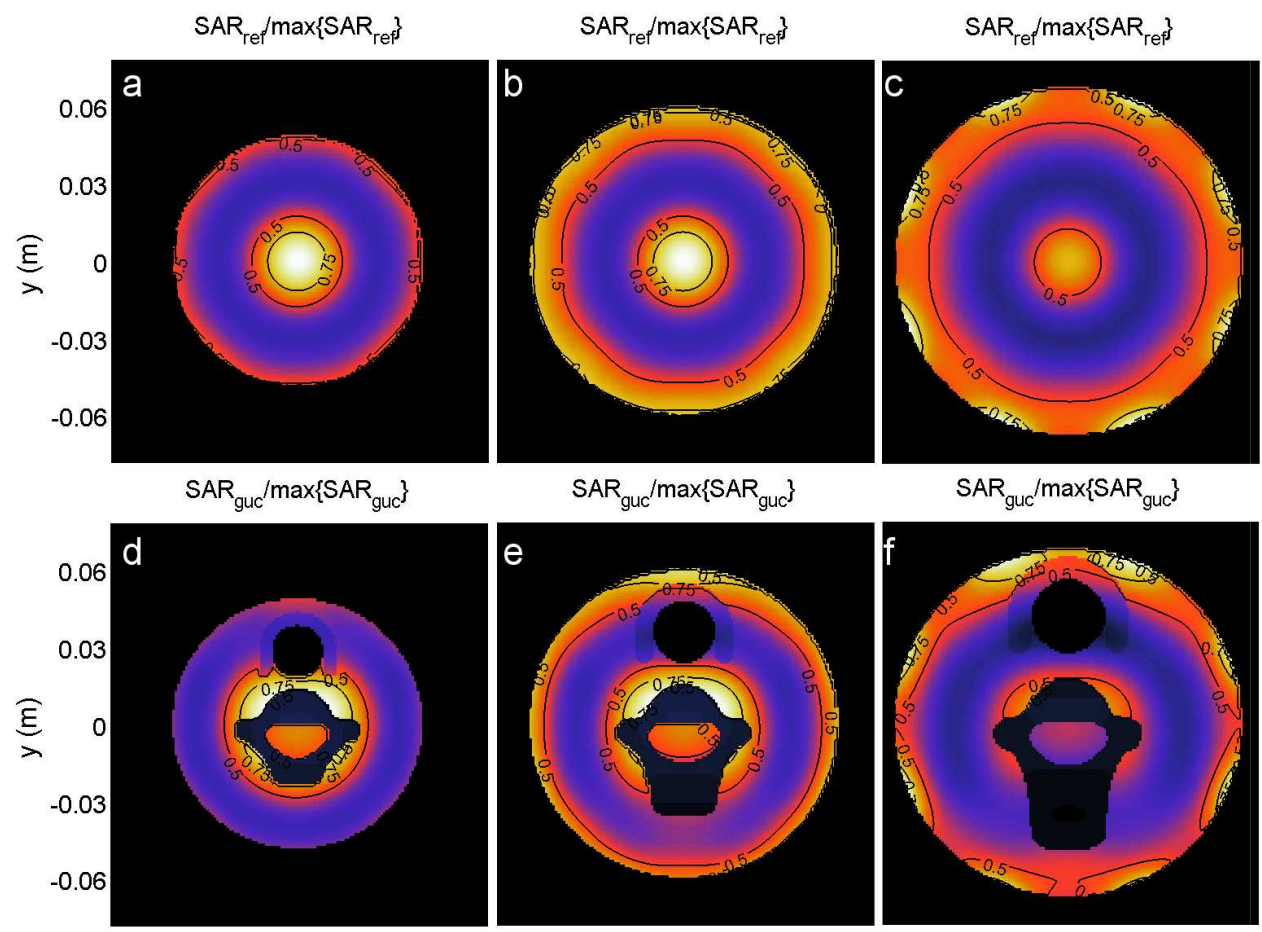

$\mathrm{SAR}_{\text {guc }} / \max \{\mathrm{SAR} \mathrm{guc}\}$
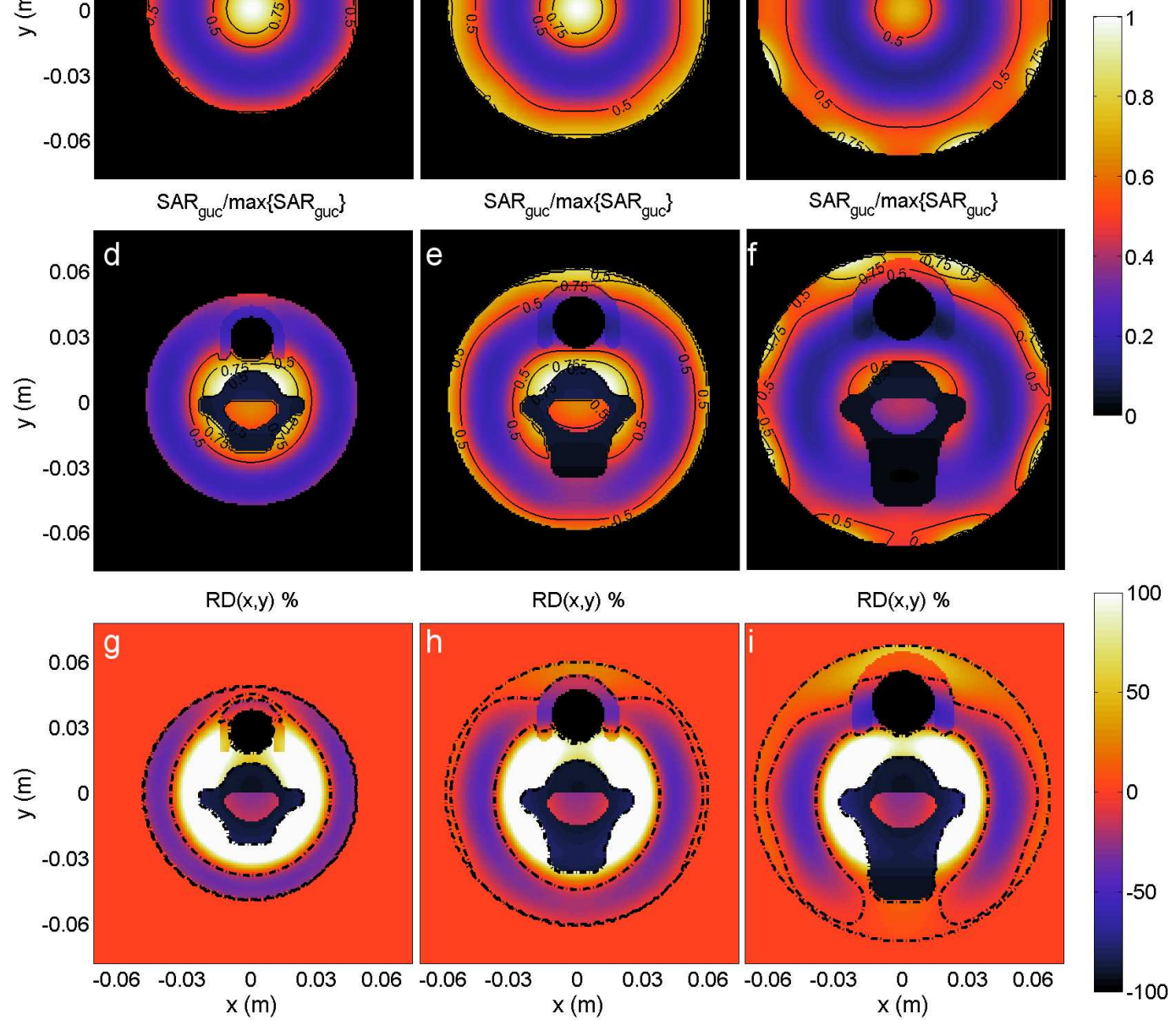

Smallest

Average

Largest

$\left(d_{\text {Neck }}=9.7 \mathrm{~cm}\right)$

$\left(d_{\text {Neck }}=12 \mathrm{~cm}\right)$

$\left(d_{N e c k}=13.5 \mathrm{~cm}\right)$

Figure 5: The SAR patterns at cross sections through the neck phantom at $\mathrm{z}=0 \mathrm{~m}$ are visualized for the muscle cylinder $\left(\mathrm{SAR}_{r e f}\right)$, the total neck setup $\left(\mathrm{SAR}_{g u c}\right)$ and their $\mathrm{RD}$ distribution for smallest, average and largest dimensions. The $50 \%$ and $75 \%$ iso-SAR contours are indicated in the $\mathrm{SAR}$ distributions and the zero RD contours are indicated in the RD distribution (-.-). 


\subsection{Influences of structure dimensions}

Figure 5 visualizes the SAR in the muscle cylinder and total neck setup and the corresponding RD distribution for the smallest, average and largest setups. It shows that the structure dimensions have a low impact on the shape of the central hotspot other than the local structure dimensions.

5 The central SAR however is highly dependent on the radius of the neck, the superficial hotspots even overrule the central hotspot for the largest dimensions setup. This is mainly due to larger path lengths in the tissue from the antennas to the center of the phantom.

Table 3 shows the $R D_{\min }, \overline{R D}, \sigma$ and $R D_{\max }$ values per geometry for setups with smallest, average and largest dimensions. From this table it is concluded that for all setups the trachea has the largest local SAR decreasing effect $\left(R D_{\min }=-100 \%\right)$ but its global effect is moderate $(\sigma \sim 23 \%)$. The global influence of the spine is high for all setups: $\sigma_{\text {spine }} \sim 60 \%$. For the smallest dimensions setup it is found that $\overline{R D} \sim 10 \%$ which can be explained by the relatively large area of increased SAR values as a result of the inclusion of the spine (see also figure 5 ).

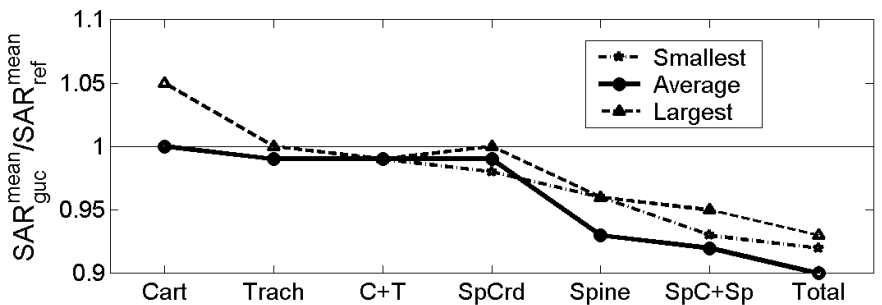

(a)

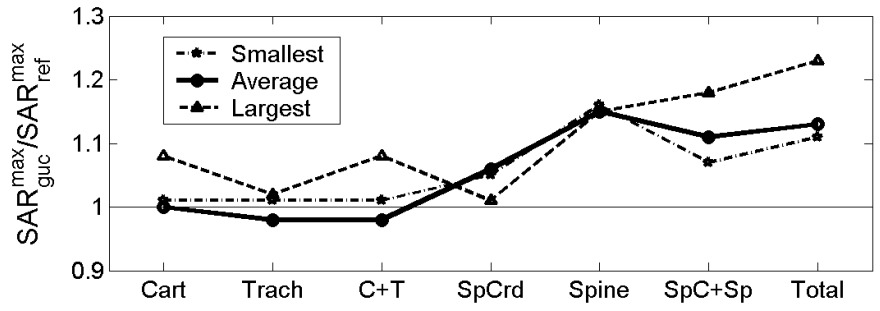

(b)

Figure 6: Mean (a) and maximum (b) SAR ratios for various structures with respect to the muscle cylinder setup per geometry for setups with smallest, average and largest dimensions

Preceding analyses are all carried out by investigating RD's calculated locally. The setups can also be compared using a more global approach, i.e. by comparing the mean and maximum SAR of the whole distribution. Figure 6 a visualizes the ratio of the average SAR levels: $\overline{S A R}_{g u c} / \overline{S A R}_{\text {ref }}$, for the three setups. Again, a large global effect of the spine is found: it decreases the overall SAR by $\sim 6 \%$. All the inserts together decrease the mean SAR on average by $\sim 7 \%$. This influence is almost independent of the dimensions of the setups. Figure $6 \mathrm{~b}$ visualizes the ratio

20 of the maximum SAR that can be found in the plane of interest. It is found that the structures increase the maximum SAR value and this increasing influence is most prominent for the setup 
with largest dimensions.

\subsection{Influence of the outer contour approximation}
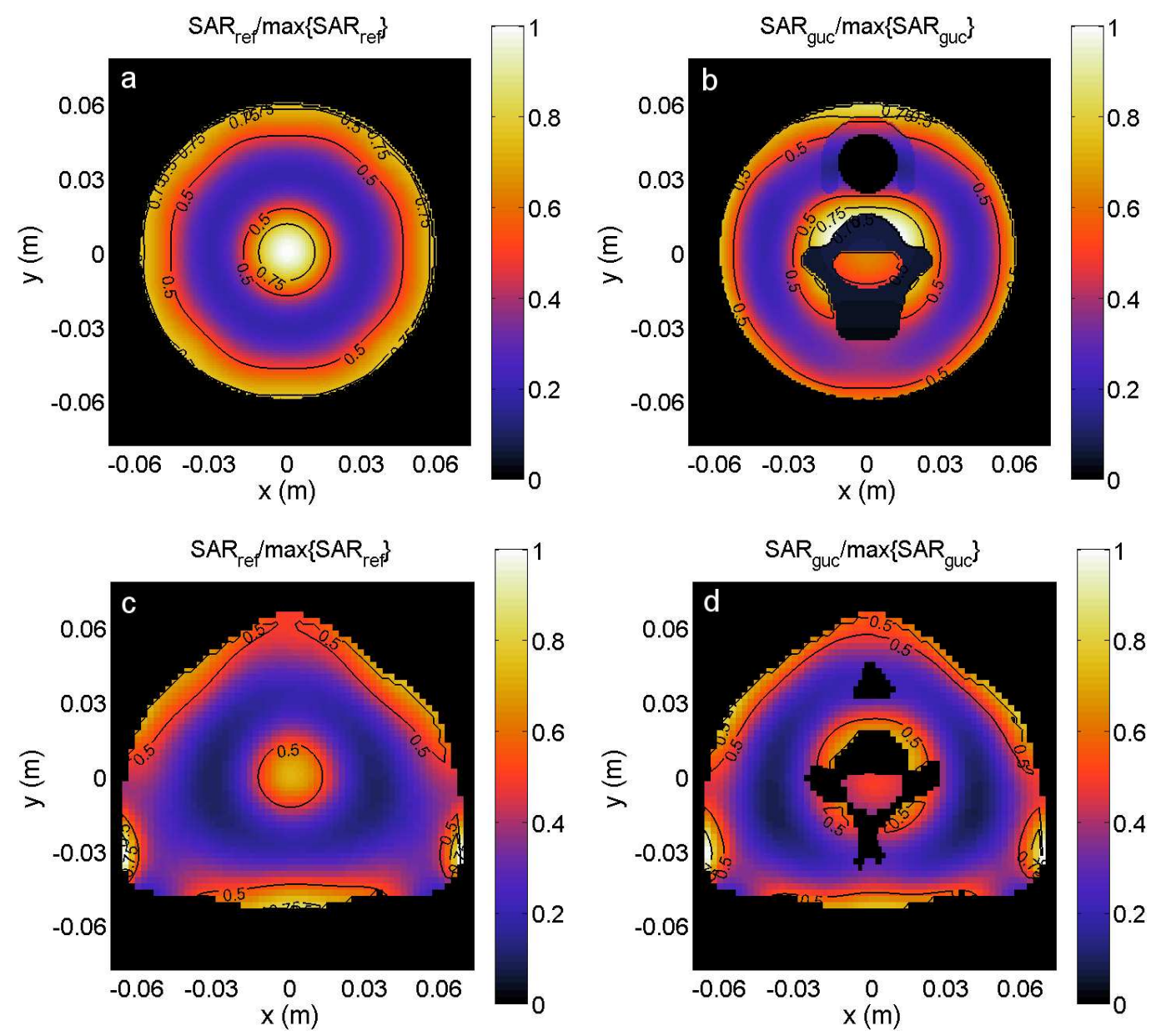

Figure 7: The SAR patterns at cross sections through the neck phantom at $\mathrm{z}=0 \mathrm{~m}$ are visualized for a) the muscle cylinder and b) the total neck setup with average dimensions and c) the muscle average anatomy setup and d) the total average anatomy-neck setup. The $50 \%$ and $75 \%$ iso-SAR contours are indicated.

In figure 7 the SAR patterns of the semi-3D cylindrical neck phantom and the semi-3D neck anatomy are visualized. Here again for all setups the central hotspot remains at its position but is

5 enlarged by the inclusion of the spine and, to a lesser extent, the spinal cord. The main difference is at the water-muscle transition that is now located closer to one of the antennas. This is in agreement with figure 5, where an increase in radius results in more superficial absorption as well. The comparison between the two setups provides some more evidence that the superficial SAR distribution is affected highly by dimensional differences but the central focus remains approximately 
intact when altering dimensions.

\section{Discussion}

\subsection{Evaluation of the materials and methods}

5 The main aim of this study is to investigate the influence on the SAR pattern of major structures in the neck, for which a semi-3D average phantom is constructed. The two main approximations for this phantom are the use of extruded 2D shapes in stead of 3D shapes and the reduction of real structure contours into elementary shapes.

The influence of the extruded 2D shape approximation is subject of further study, however some remarks on the accuracy of the SAR distributions can be made. Firstly, the extends of the spine are used for the determination of the average dimensions for the average setup, i.e. the gaps between the (spinous process of the) cervical vertebras is "filled" with bone material. This is a fair approximation since the fields are axially polarized and because the gaps between the cervical vertebra's are approximately $1 \mathrm{~cm}(=\lambda / 9$ in muscle). Therefore the wave interacts with the independent vertebra's as if it is one solid obstacle (with an effective permittivity and conductivity). Secondly, the trachea and spinal cord are approximately vertical in the region of interest and can therefore be approximated by 2D structures (see figure 1). Other structures, located outside the region of interest could influence the SAR pattern due to field rotations. Most of them, however, are located far from the region under consideration.

The influence of the reduction into elementary shapes, in stead of the use of the actual patient contours, on the central SAR focus is small. However from figure 7 it is found that the superficial SAR is increased for a $2 \mathrm{D}$ anatomy phantom as a result of the difference in skin contour. Note though that the setup that is used for the comparison is constructed from one CT slice of one closeto-average patient. The finding, however, is in agreement with the trend from the size investigation, 25 i.e. the SAR in a total neck setup is increased in areas located just over $6 \mathrm{~cm}(=2 / 3 \lambda$ in muscle) from the center (see the RD distributions in figure 5). From this comparison it is concluded that the shapes of the SAR distributions, and therefore the trends of the findings, only moderately change as a result of the outer contour approximation. This conclusion might also be of importance with respect to quality assurance measurements since these are usually carried out utilizing cylindrical 30 setups.

\subsubsection{Influence of dielectric properties, voxelsizes and a subcutaneous fat layer}

In literature, the variations with respect to the permittivity and conductivity values of table 1 are approximately $25 \%$ [14] [15] [16], where the conductivity has a tendency to be somewhat higher compared to the values in the table. From Van de Kamer et al. [17] it is found that the influence on

35 the mean SAR value of these parameters is about $10 \%$ in an actual patient anatomy. If, however, accurate dielectric parameters for fat are utilized this value is about $6 \%$. Since in the neck no fat is modelled we estimate the accuracy of the SAR distribution at this $6 \%$. The worst case estimation 
for the RD distribution therefore is $12 \%$ global difference. This estimation is useful only for global values: it can not be used for local influences.

Calculations for every structure and average dimensions at a lower resolution $(2.5 \mathrm{~mm}$ for $\Delta \mathrm{x}$ and $\Delta \mathrm{y}$ and $1 \mathrm{~mm}-5 \mathrm{~mm}$ for $\Delta \mathrm{z}$ ) are carried out in order to assess the influence of the results 5 on the grid size. Comparing the results of the $2.5 \mathrm{~mm}$ with the $1 \mathrm{~mm}$ resolution we find for the differences: $\Delta \overline{R D}<2 \%, \Delta R D_{\max }<11 \%$ and $\Delta S A R_{50 \%}<0.2 \mathrm{~cm}^{2}(1.4 \%)$. This means that the $50 \%$ iso-SAR pattern is almost independent of this gridsize change and that the influence on the $\mathrm{RD}$ values is small.

The influence of the subcutaneous fat layer is neglected in this study. To investigate the

10 effect of such a layer we carried out calculations with a $3 \mathrm{~mm}$ thick fat layer at the transition between the muscle and water environment. The conclusion from these calculations are that, due to increased reflections, the amount of SAR is decreased : $\Delta \overline{R D}=-12 \%, \Delta R D_{\max }=-18 \%$ and $\Delta S A R_{50 \%}=-0.3 \mathrm{~cm}^{2}$, but the pattern remains intact for all structures and thus $\sigma$ remains approximately equal $(\Delta \sigma=-0.3 \%)$. The total amount of SAR in the cylinder is reduced $\sim 14 \%$

15 after inclusion of this fat layer.

\subsection{SAR and RD distributions: trends and clinical translation}

With respect to the clinical translation this investigation forms a first step into the systematic investigation of the parameters that influence the quality of an HT treatment of H\&N tumours. Next steps will consist of investigations into 3D-anatomy distorting influences and the influences of the applicator, i.e. antenna ring(s) and waterbolus. Subsequently the main treatment influencing parameters can be identified and used as an aim for the design of an applicator.

For the muscle calculations it is found that a $\lambda / 3$ in diameter central hotspot is created by eight circumferential placed dipole antennas. This is in agreement with literature values for the central hotspot in muscle [19] [22].

The influence of the trachea and spine on the SAR pattern is found to be large. The influence of the trachea is mainly local but the spine has a more global influence, i.e. it spreads the central hotspot from $\lambda / 3$ in diameter to approximately $2 / 3 \lambda$. This value $(2 / 3 \lambda)$ is in agreement to calculations, carried out by Guy et al. [19], at $915 \mathrm{MHz}$ using an anatomy, which are verified with measurements. The location of the first region of low SAR, seen from the center, is identical to the results of Guy [19] et al. as well: $2 / 3 \lambda$ for the muscle cylinder, $\lambda / 2$ for the layered bone-muscle-fat cylinder. The difference lies in the fact that for this work a plane wave illumination from four sides is used and therefore the superficial absorption by closely positioned antennas is not modelled. The resemblance of the central phenomena does agree with the finding that the shape of the structures

35 is of moderate importance but that its position in the SAR pattern and its dielectric contrast are of high importance.

$433 \mathrm{MHz}$ is used as operating frequency in this study, which has a major effect on the results. The pattern in the neck is highly determined by the wavelength and the dielectric properties of the 40 tissues, thus by the operating frequency. This study indicates that $433 \mathrm{MHz}$ is a suitable choice 
since its penetration depth is borderline sufficient for large structures but still phase and amplitude steering are possible due to a single central hotspot in all setups. This finding is concluded as well from SAR measurements in muscle phantoms at $400 \mathrm{MHz}$ and $915 \mathrm{MHz}$ [23]. This conclusion however needs confirmation by calculations with a 3D anatomy since 1) the influence of fields reflected

5 at higher and lower structures and at oblique transitions are not included in this study and 2) a symmetrical setup is used for the calculations.

With respect to the design of the future clinical $H \& N$ applicator we found little evidence of disturbing effects that would restrict any specific applicator design. We found a minor indication

10 that the only avoidable, undesired, increase of the SAR is located at the trachea. Further, no evidence is found of a limiting change of the SAR in the spinal cord, which is a highly relevant issue because of the potential of neurotoxicity. This study indicates that a cylindrical muscle phantom with a bony spine insert is a suitable configuration for fast and simple inter-antenna performance comparisons and provides good opportunities for experimental verification.

\section{Conclusions}

From this study it is found that the anatomy at $433 \mathrm{MHz}$, and especially the conductivity distribution, has a major impact on the SAR distribution in the neck: up to $\sim 11 \%$ global (mean) relative difference and a standard deviation from the mean of up to $\sim 80 \%$ with respect to a muscle cylinder. Central, low in conductivity, structures cause the $50 \%$ iso-SAR contour to be widened

20 and have a local influence of up to $\sim 270 \%$. The focal region is changed from a circular to a more donut shape following the conductivity distribution in the neck. This effect is almost independent of the dimensions of the neck and the outer contours of the structures. The $50 \%$ and $75 \%$ iso-SAR contours remain identical for changing dimensions up to a threshold from which the path lengths in tissue from the antennas towards the center of the neck become to large with respect to the 25 penetration depth.

The results in this study are obtained with use of an extruded semi-2D elementary shape-fit phantom. Further study with 3D setups is necessary to investigate the influence of 3D structures on the SAR pattern. This study however forms a first step towards the understanding of the complex influences of the various parameters on the SAR pattern in the head and neck and the design of a specific EM-applicator for heating $\mathrm{H} \& \mathrm{~N}$ tumours.

\section{Acknowledgements}

This work is financially supported by the Dutch Cancer Society, grant DDHK 2003-2855. The authors further would like to thank Professor A.P.M. Zwamborn for his contribution to the discussion. 


\section{References}

[1] Valdagni R, Liu F-F and Kapp S. Important prognostic factors influencing outcome of combined radiation and hyperthermia. Int. J. Radiat. Oncol. Biol. Phys. 1988; 15: 959-972.

[2] Valdagni R and Amichetti M. Report of long-term follow-up in a randomized trial comparing radiation therapy and radiation therapy plus hyperthermia to metastatic lymphnodes in stage IV head and neck patients. Int. J. Radiat. Oncol. Biol. Phys. 1993; 28: 163-169.

[3] Wust P, Seebas M, Nadobny J, Deuflhard P, Mönich G, Felix R. Simulation studies promote technological development of radiofrequency phased array hyperthermia. Int. J. Hyperthermia 1996; 12: 477-98.

[4] Jacobsen S, Melands $\varnothing$ F. The concept of using multifrequency energy transmission to reduce hot-spots during deep-body hyperthermia. Ann. Biom. Eng. 2002; 30: 1-10.

[5] Möller TB, Reif E. Pocket atlas of cross-sectional anatomy: CT and MRI, Volume 1: Head, Neck, Spine and Joints. 1994, Georg Thieme Verlag Stuttgart, New York.

[6] SEMCAD: the Simulation platform for EMC, Antenna Design and Dosimetry Smith and Partner Engineering AG http://www.semcad.com

[7] Jouvie F, Bolomey JC, Gaboriaud G. Discussion of the capabilities of microwave phased arrays for hyperthermia treatment of neck tumours. IEEE Trans. Micr. Theor. Tech. 1986; $34: 495-501$.

20 [8] Balanis CA. Antenna theory: analysis and design, second edition. 1997, John Wiley \& Sons inc., New York, Chichester, Brisbane, Toronto, Singapore. ISBN 0-471-59268-4, Chapter 4.

[9] Taflove A. Computational electrodynamics: The Finite-Difference Time-Domain Method. 1995, Artech House; 685 Canton Street; Norwood, MA 02062. ISBN: 0-89006-792-9, Chapter $3 \& 6$.

25 [10] IFAC-CNR, Florence (Italy), 1997-2002, http://niremf.iroe.fi.cnr.it/tissprop/htmlclie/htmlclie.htm, Application prepared by Andreuccetti, D., Fossi, R. and Petrucci, C.

[11] Gabriel S, Lau RW and Gabriel C. The dielectric properties of biological tissues III: Parametric models for the dielectric spectrum of tissues. Phys. Med. Biol. 1996; 41: 2271-93.

30 [12] Stogryn A. Equations for Calculating the Dielectric Constant of Saline Water (Correspondence). IEEE Trans. Micr. Theor. Tech. 1971; 19: 733-36.

[13] Lagendijk JJW, Van den Berg PM, Hand JW, Uzunoglu NK, Sheppard R, Bach Andersen J, Bardati F, Bolomey JC. Treatment planning and modelling in hyperthermia, a task group report. 1992, Postgraduate school of medical physics, II University of Rome. 
[14] Gabriel C, Gabriel S and Corthout E. The dielectric properties of biological tissues I: Literature survey. Phys. Med. Biol. 1996; 41: 2231-49.

[15] Gabriel S, Lau RW and Gabriel C. The dielectric properties of biological tissues II: Measurements in the frequency range $10 \mathrm{~Hz}$ to $20 \mathrm{GHz}$. Phys. Med. Biol. 1996; 41: 2251-69.

5 [16] Field SB, Hand JW. An introduction to the practical aspects of clinical hyperthermia. 1990. Taylor \& Francis Ltd., 4 John st., London WC1N2ET.

[17] Van de Kamer JB, Van Wieringen N, De Leeuw AAC, and Lagendijk JJW. The significance of accurate dielectric tissue data for hyperthermia treatment planning. Int. J. Hyperthermia 2001; 17: 123-42.

10 [18] Hertel TW, Smith GS. On the convergence of common FDTD feed models for antennas. IEEE Trans. Ant. Prop. 2003; 51: 1771-79.

[19] Guy AW, Chou CK, Luk KH. 915-MHz phased-array system for treating tumors in cylindrical structures. IEEE Trans. Microwave Theory Tech. 1986; 34: 502-07.

[20] van Rhoon GC, Visser AG, van den Berg PM, Reinhold HS. Evaluation of ring capacitor plates for regional deep heating. Int. J. Hyperthermia 1988; 4: 133-42.

[21] van Rhoon GC, Sowinski MJ, van den Berg PM, Visser AG, Reinhold HS. A ring capacitor applicator in hyperthermia: energy distributions in a fat-muscle layered model for different ring electrode configurations. Int. J. Radiation Oncology Biol. Phys. 1990; 18: 77-85.

[22] van Rhoon GC, van der Heuvel DJ, Ameziane A, Rietveld PJM, Volenec K, van der Zee J. Characterization of the SAR-distribution of the Sigma-60 applicator for regional hyperthermia using a Schottky diode sheet. Int. J. Hyperthermia 2003; 6: 642-54.

[23] Gross EJ, Ceetas TC, Stauffer PR, Liu RL, Lumori MLD. Experimental assessment of phasedarray heating of neck tumours. Int. J. Hyperthermia. 1990; 6: 453-74. 